\title{
Eicosapentaenoic Acid Monoacylglyceride
}

National Cancer Institute

\section{Source}

National Cancer Institute. Eicosapentaenoic Acid Monoacylglyceride. NCI Thesaurus. Code C158515.

An eicosapentaenoic acid derivative with potential antineoplastic activities. Upon oral administration, eicosapentaenoic acid monoacylglyceride (MAG-EPA) may inhibit epidermal growth factor receptor (EGFR), vascular endothelial growth factor receptor (VEGFR) and serine/threonine protein kinase AKT (protein kinase B)-mediated signaling pathways and reduce vascular endothelial growth factor (VEGF) and hypoxia-inducible factor 1-alpha (HIF1-alpha) expression levels in tumor cells. This may promote apoptosis and inhibit tumor cell growth in cells expressing EGFR, AKT, and VEGFR. MAG-EPA may also inhibit androgen-stimulated tumor cell growth and repress androgenic induction of prostate-specific antigen (PSA) expression. PSA, a tumor associated antigen (TAA), is expressed by prostate epithelial cells and is overexpressed in prostate cancer. The AKT signaling pathway is often dysregulated in cancer and is associated with tumor cell proliferation, survival and migration. EGFR and VGFR are receptor tyrosine kinases that are mutated or upregulated in many tumor cell types and play a key role in tumor cell proliferation and tumor vascularization. 